\title{
Investir dans la formation des personnes enseignantes associées pour faire du stage en enseignement un instrument de développement professionnel \\ Investing in supervising-teacher training to make teaching practicums a professional development tool Invertir en la formación de los maestros asociados con el fin de convertir el período de prácticas en enseñanza en un instrumento de desarrollo profesional
}

\author{
Jean-François Desbiens, Cecilia Borges et Carlo Spallanzani
}

Volume 37, numéro 1, printemps 2009

La supervision pédagogique en enseignement de l'éducation physique

URI : https://id.erudit.org/iderudit/037650ar

DOI : https://doi.org/10.7202/037650ar

Aller au sommaire du numéro

\section{Éditeur(s)}

Association canadienne d'éducation de langue française

ISSN

0849-1089 (imprimé)

1916-8659 (numérique)

\section{Découvrir la revue}

Citer cet article

Desbiens, J.-F., Borges, C. \& Spallanzani, C. (2009). Investir dans la formation des personnes enseignantes associées pour faire du stage en enseignement un instrument de développement professionnel. Éducation et francophonie, 37(1), 6-25. https://doi.org/10.7202/037650ar

\section{Résumé de l'article}

L'augmentation notable du nombre d'heures consacré au stage est l'une des conséquences directes de la récente vague de réformes des programmes de formation initiale à l'enseignement constatée en Amérique du Nord et ailleurs dans le monde. S'inscrivant dans une visée de professionnalisation du métier, ces réformes ont accru, sans vraiment la consacrer, la place occupée par la personne enseignante associée (PEA) dans ce processus. À titre de formatrice, cette personne, dont l'influence perçue sur le développement professionnel des futurs enseignants pèse déjà lourdement, ne reçoit toujours pas toute la considération qui lui revient. Cette influence suscite toutefois des questions relatives à la qualité des expériences d'apprentissage du métier fournies aux stagiaires durant les stages. Elle soulève en outre des préoccupations relativement à la sélection des PEA ainsi qu'à leur formation en matière de supervision pédagogique, un aspect encore largement négligé dans la formation des futurs enseignants. Cet article consiste en une revue de la documentation scientifique centrée sur le stage comme moment de formation et sur les PEA vues comme des acteurs centraux remplissant des fonctions particulières, nécessitant des compétences à la fois distinctes et complémentaires de celles qui sont requises pour exercer le métier d'enseignant. Les auteurs vont soutenir l'idée qu'investir davantage de ressources dans la formation de ces personnes constitue une façon d'élever le potentiel formateur du stage en enseignement et, ultimement, d'améliorer la compétence du corps enseignant dans son ensemble.
Tous droits réservés @ Association canadienne d'éducation de langue française, Ce document est protégé par la loi sur le droit d’auteur. L’utilisation des 2009 services d’Érudit (y compris la reproduction) est assujettie à sa politique d'utilisation que vous pouvez consulter en ligne.

https://apropos.erudit.org/fr/usagers/politique-dutilisation/ 


\section{Investir dans la formation des personnes enseignantes associées pour faire du stage en enseignement un instru- ment de développement professionnel}

Jean-François DESBIENS

Université de Sherbrooke, GRIEFPAP, CRIFPE

\section{Cecilia BORGES}

Université de Montréal, GRIEFPAP, CRIFPE

Carlo SPALLANZANI

Université de Sherbrooke, GRIEFPAP, CRIE, CRIFPE

\section{RÉSUMÉ}

L'augmentation notable du nombre d'heures consacré au stage est l'une des conséquences directes de la récente vague de réformes des programmes de formation initiale à l'enseignement constatée en Amérique du Nord et ailleurs dans le monde. S'inscrivant dans une visée de professionnalisation du métier, ces réformes ont accru, sans vraiment la consacrer, la place occupée par la personne enseignante associée (PEA) dans ce processus. À titre de formatrice, cette personne, dont l'influence perçue sur le développement professionnel des futurs enseignants pèse 
déjà lourdement, ne reçoit toujours pas toute la considération qui lui revient. Cette influence suscite toutefois des questions relatives à la qualité des expériences d'apprentissage du métier fournies aux stagiaires durant les stages. Elle soulève en outre des préoccupations relativement à la sélection des PEA ainsi qu'à leur formation en matière de supervision pédagogique, un aspect encore largement négligé dans la formation des futurs enseignants. Cet article consiste en une revue de la documentation scientifique centrée sur le stage comme moment de formation et sur les PEA vues comme des acteurs centraux remplissant des fonctions particulières, nécessitant des compétences à la fois distinctes et complémentaires de celles qui sont requises pour exercer le métier d'enseignant. Les auteurs vont soutenir l'idée qu'investir davantage de ressources dans la formation de ces personnes constitue une façon d'élever le potentiel formateur du stage en enseignement et, ultimement, d'améliorer la compétence du corps enseignant dans son ensemble.

\section{ABSTRACT}

\section{Investing in supervising-teacher training to make teaching practicums a professional development tool}

Jean-François DESBIENS, University of Sherbrooke, GRIEFPAP, CRIFPE

Cecilia BORGES, University of Montréal, GRIEFPAP, CRIFPE

Carlo SPALLANZANI, University of Sherbrooke, GRIEFPAP, CRIE, CRIFPE

The notable increase in the number of hours devoted to teaching practicums is a direct consequence of the recent wave of reforms to initial teacher training programs in North America and elsewhere in the world. These reforms, aimed at professionalizing teaching, granted more importance to the supervising teacher (ST) without really consecrating the role. Although the influence these teachers have on the professional development of future teachers is already perceived as very important, they do not always receive the consideration they deserve. However, this influence raises questions about the quality of the learning experiences student teachers are receiving during practicums, and concerns about the selection of ST and their pedagogical supervision training, an aspect that is still largely neglected in the training of future teachers. This article consists of a review of the scientific literature about teacher training, with ST being the main actors who fulfil specific functions requiring skills that are both distinct from and complementary to regular teaching skills. The authors support the idea that investing more resources in ST training is one way to raise the training potential of the practicum and ultimately improve the overall competence of the teaching corps. 


\section{RESUMEN}

\section{Invertir en la formación de los maestros asociados con el fin de convertir el período de prácticas en enseñanza en un instrumento de desarrollo profesional}

Jean-François DESBIENS, Universidad de Sherbrooke, GRIEFPAP, CRIFPE

Cecilia BORGES, Universidad de Montreal, GRIEFPAP, CRIFPE

Carlo SPALLANZANI, Universidad de Sherbrooke, GRIEFPAP, CRIE, CRIFPE

El notable aumento del número de horas consagradas al período de prácticas es una de las consecuencias directas de la reciente ola de reformas de los programas de formación inicial de docentes constatadas en America del norte y en otras partes del mundo. Inscritas en la intención de profesionalizar dicho oficio, esas reformas han incrementado, sin haber logrado consagrar, el lugar que ocupa el individuo que funge como maestro asociado (IMA) en dicho proceso. En tanto que formador, cuya influencia sobre el desarrollo profesional de los futuros maestros es bastante importante, el individuo que funge como maestro asociado no siempre recibe la consideración que merece. No obstante, dicha influencia suscita cuestiones relacionadas con la calidad de las experiencias de aprendizaje del oficio que se ofrecen a los practicantes durante el período de prácticas. Además, plantea cuestiones concernientes a la selección del IMA y a su formación en materia de supervisión pedagógica, un aspecto aun ampliamente desatendido en la formación de los futuros maestros. Este artículo es una revista de la documentación científica centrada en el período de prácticas en tanto que etapa de formación y sobre el IMA, actor principal que ocupa funciones que necesitan habilidades a la vez específicas y complementarias de las que se requiere para ejercer el oficio de maestro. Los autores apoyan la idea que una mayor inversión de recursos en la formación estos últimos constituye una manera de incrementar el potencial formador del período de prácticas en docencia y, finalmente, de aumentar las habilidades de todo el cuerpo profesoral.

\section{Introduction}

La publication en 2001 par le ministère de l'Éducation du Québec (MEQ) ${ }^{1}$ (Martinet, Gauthier et Raymond, 2001) de nouvelles orientations pour la formation à l'enseignement a souligné l'importance des stages supervisés dans le développement des compétences en enseignement, peu importe les spécialités. Cette tendance,

1. Le ministère de l'Éducation du Québec est aujourd'hui dénommé ministère de l'Éducation, du Loisir et du Sport (MELS) 
observable dans plusieurs pays et signalée dès la fin des années 1990 par Koster, Korthagen et Wubbels (1998), confirme dans les faits l'importance croissante de la fonction de personne enseignante associée (PEA) (aussi appelée conseiller pédagogique, maître de stage, maître associé, etc.) en formation initiale en enseignement. Au Québec, avec un minimum de 700 heures de stage étalées sur les quatre années de la formation initiale, l'abolition de la période de probation² menant à l'obtention du brevet en enseignement (MEQ, 1995) et les départs massifs à la retraite d'enseignants chevronnés (COFPE, 2005), cette tendance revêt toutefois une signification bien particulière, parce qu'elle soulève inévitablement un ensemble de questions touchant la supervision des stagiaires par les PEA du primaire et du secondaire, la sélection de ces dernières ainsi que l'adéquation de leur préparation à exercer cette fonction professionnelle.

Cet article introductif à portée générale est divisé en quatre parties. Dans la première, une réflexion sur le statut du stage en formation initiale à l'enseignement est menée. Cette réflexion aura le Québec pour principal point d'ancrage. Dans la seconde et la troisième partie, le regard est porté sur la personne enseignante associée (PEA), dont l'importance est devenue centrale en raison des nouveaux cadres de la formation des maîtres, puis sur la formation à l'exercice de la supervision dont elle bénéficie. Dans la quatrième partie, la nécessité de mieux articuler les compétences à définir pour la supervision des stagiaires par les personnes enseignantes associées aux fonctions qu'elles doivent remplir est soulevée.

\section{Quel statut donner au stage en formation initiale à l'enseignement?}

Le stage en enseignement est présenté comme une occasion de vivre la «complexité de l'acte d'enseigner» (Ministère de l'Éducation du Québec [MEQ], 1994a, p. 20). Il consiste, pour le ministère de l'Éducation du Québec (MEQ), (1994a, p. 3) en «un ensemble d'activités éducatives supervisées par l'université en collaboration avec le milieu scolaire. Par un entraînement systématique et réfléchi, il permet au futur enseignant de développer sa capacité d'intégrer et de mettre en application, dans un milieu réel, les principes qui fonderont sa pratique quotidienne.»

2. À la suite des recommandations du rapport Parent (Commission royale d'enquête sur l'enseignement dans la province de Québec, 1963), la responsabilité de la formation à l'enseignement au Québec est confiée aux universités plutôt qu'aux écoles normales. Ce faisant, on a voulu lui accorder une plus grande importance (Conseil supérieur de l'éducation, 2004). À partir de 1970, il a été jugé nécessaire que le nouvel enseignant qui avait obtenu un permis temporaire au terme de sa formation universitaire soit soumis à une période probatoire avant d'obtenir son brevet définitif (Conseil supérieur de l'éducation, 2004). Durant cette période de deux ans, le nouvel enseignant devait prouver sa compétence. Le milieu d'accueil constituait un comité probatoire pour chaque nouvel enseignant. Ce comité était composé d'un membre de la direction de l'école et d'un enseignant breveté (Thibeault, 1994). La commission scolaire avait la responsabilité de mettre à la disposition des candidats les documents relatifs à la période probatoire et d'attester en fin de compte la réussite ou l'échec de la probation. De son côté, I'enseignant était tenu de tenir à jour le cumul de son temps d'enseignement et de demander à la commission scolaire de lui délivrer une attestation de réussite ou un avis d'échec. 
D’abord considéré durant les années 1970

à 1990 comme un temps d'application des savoirs professionnels appris en institutions de formation des maîtres dans la logique de ce que Schön (1994) a appelé la « rationalité technique » ou de ce que Martinet, Gauthier et Raymond (2001) ont nommé l'« utopie scientiste ", le stage est maintenant pensé dans la perspective du maître professionnel.
L'importance maintenant accordée au stage en enseignement en matière de durée, d'allocation de crédits de formation et de positionnement au sein des programmes de formation est, nous semble-t-il, l'indication d'un changement de perspective dans l'ensemble de la formation des maîtres. D'abord considéré durant les années 1970 à 1990 comme un temps d'application des savoirs professionnels appris en institutions de formation des maîtres dans la logique de ce que Schön (1994) a appelé la « rationalité technique » ou de ce que Martinet, Gauthier et Raymond (2001) ont nommé l' «utopie scientiste», le stage est maintenant pensé dans la perspective du maître professionnel. Cela correspond « [...] à la redécouverte de la valeur et de l'importance de la pratique professionnelle comme source du savoir d'expérience, considéré lui-même comme fondement de la compétence professionnelle des enseignants de métier» (Tardif, 2006, p. 13). Tardif (2006) insiste pour dire qu'il ne s'agit pas ici de nier l'importance des connaissances pédagogiques, didactiques et disciplinaires, mais plutôt de les mettre au service de l'apprentissage de la pratique professionnelle. D'ailleurs, certains travaux menés en éducation physique (Brawdy et Byra, 1995) suggèrent qu'une façon efficace d'aider les futurs maîtres à développer des comportements d'enseignement appropriés est de combiner très tôt l'expérience pratique de l'enseignement en laboratoire, de même qu'en milieu scolaire, avec les cours suivis en formation des maîtres.

Dans ce cadre, le stage est présenté comme un temps de formation qui favorise une familiarisation concrète avec les dynamiques complexes de l'école et de la classe, la construction de capacités d'action professionnelle (Organisation pour la coopération et le développement économiques [OCDE], 2005) et la réflexion sur les précédents éléments s'inscrivant en alternance ainsi qu'en continuité avec d'autres temps de formation.

Constatant l'incomplétude des cadres d'analyse de l'apprentissage de l'enseignement, Munby, Russell et Marten (2001) ont insisté sur le fait que la connaissance de la façon dont agissent les bons enseignants ou celle du contenu de leur savoir professionnel ne suffisaient pas pour savoir comment ils en étaient venus à penser et à agir comme ils le font lorsqu'ils enseignent. S'appuyant sur les travaux de Carter (1990), ils ont soutenu que le savoir des enseignants n'est pas largement abstrait et propositionnel et qu'il ne peut non plus être formalisé en un ensemble d'habiletés ou de réponses préfabriquées à des problèmes précis. Ce savoir est plutôt de nature expérientielle, procédurale, située et particularisée. Il est contextualisé et incorporé dans les pratiques d'où il émerge et dans lesquelles il est utilisé.

Se référant ensuite à Connelly et Clandinin (1990), Munby et al. (2001) ont rappelé la part biographique et historique du savoir des enseignants. Cette référence n'est pas sans conséquence pour la formation à l'enseignement, puisque, comme le mentionne Raymond (2006, p. 239), « [l]a formation professionnelle à l'enseignement se réalise dans un domaine auquel les aspirants ont été amplement socialisés, pour lequel ils estiment déjà posséder les principales compétences et dans lequel ils s'attendent à réussir sans s'engager dans une préparation exigeante ». Cette importante part de l'histoire des enseignants est particulièrement prégnante chez certains candidats des programmes de formation à l'enseignement de l'éducation physique 
Le stage est perçu comme l'une de ces expériences qui marquent l'individu tant par la durée que par I'intensité de ce qui y est vécu (Tardif et Lessard, 1999). Pour bon nombre de stagiaires, c'est la dimension la plus signifiante du programme de formation des maîtres, le moment tant attendu d'apprendre à enseigner (Banville, 2002; Jordan, Phillips et Brown, 2004; Keller et Grossman, 1994; Knowles et Sudzina, 1994).

qui croient que leurs expériences dans les secteurs de l'entraînement sportif, du conditionnement physique et de l'animation d'activités physiques auprès d'équipes sportives ou de jeunes dans des camps de vacances les ont déjà amplement préparés à exercer ce métier en contexte scolaire.

Munby et al. (2001) ont enfin défendu l'idée que le savoir-enseigner ne peut être caractérisé indépendamment de la façon dont il est appris et utilisé. Ce savoir dépendrait à la fois des dimensions matérielles, physiques et sociales de l'environnement de travail des enseignants, ce qui pose inévitablement la question de son transfert à d'autres contextes, de ses limites et, ultimement, celle plus délicate, de la portée véritable du stage comme activité de formation préparatoire à l'exercice de la fonction enseignante. Ainsi, retenons que le savoir-enseigner prend sa source dans l'histoire personnelle des enseignants, qu'il est aussi en partie pratique et que cette part ne peut être apprise que par la pratique. Cela pose bien entendu le problème du degré de contrôle ou de l'emprise réelle du formateur universitaire de maîtres sur l'ensemble du processus de formation à l'enseignement.

Cette centralité nouvelle des stages dans l'apprentissage de connaissances pratiques conduisant à des actions pédagogico-didactiques mieux adaptées semble de plus en plus admise et fondée sur des travaux de recherche sur la cognition (Talvitie, Peltokallio et Männistö, 2000). Elle rejoint le point de vue émis par Masciotra (2007) selon qui l'agir compétent s'exerce et se développe en situation, de même que celui de Martinet et al. (2001) pour qui la compétence se déploie en contexte professionnel. Le stage constitue donc une occasion de rencontrer de nombreuses situations typiques ou non qui sont autant de stimuli au développement d'instruments de compréhension des situations de classe et de soi comme enseignant. En retour, ces instruments enrichissent les pratiques d'enseignement subséquentes ainsi que la réflexion professionnelle (Schmidt et Knowles, 1994) et contribuent à l'édification progressive d'une jurisprudence privée, d'un savoir d'expérience (Gauthier, Desbiens, Malo, Martineau et Simard, 1997), c'est-à-dire d'un savoir composite parce que fait d'éléments de nature différente, composite aussi parce que ces éléments proviennent de lieux différents et que leur intégration procède selon des logiques différentes (Tardif et Lessard, 1999).

Le stage est perçu comme l'une de ces expériences qui marquent l'individu tant par la durée que par l'intensité de ce qui y est vécu (Tardif et Lessard, 1999). Pour bon nombre de stagiaires, c'est la dimension la plus signifiante du programme de formation des maîtres, le moment tant attendu d'apprendre à enseigner (Banville, 2002; Jordan, Phillips et Brown, 2004; Keller et Grossman, 1994; Knowles et Sudzina, 1994). Toutefois, pour bien d'autres, c'est une période extrêmement angoissante, un long test d'endurance marqué par de la confusion sur les plans de la définition des attentes à satisfaire, des rôles à assumer et des fonctions à exercer (Kosnic, sous presse), peut-être parce qu'ils en ont souvent mésestimé la complexité. La documentation consultée (Chaliès et Durand, 2000; Holvast, Wubbels et Brekelmans, 1993) indique également que les expériences de stage sont souvent peu cohérentes parce qu'elles poursuivent des objectifs imprécis, qu'elles sont mal cadrées théoriquement ou qu'elles présentent des lacunes sur les plans de la planification du travail et de 
l'encadrement fourni par les milieux d'accueil. S'il n'existe qu'une façon d'initier le stagiaire aux divers aspects du métier, le stage peut ne devenir qu'une façon de «lessiver » les apprentissages réalisés dans son programme de formation et de perdre de vue les finalités éducatives et sociales poursuivies à travers les gestes quotidiens de l'enseignant.

Les éléments qui précèdent mettent en relief le rôle central du stage dans l'édification du savoir-enseigner, tout en attirant l'attention sur le fait qu'en l'absence de conditions propices à l'apprentissage de l'enseignement cette activité n'aura peut-être pas les retombées attendues. Contrairement à une position souvent formulée, la capacité d'enseigner n'est pas innée (Gauthier et al., 1997) : elle peut s'apprendre, se développer, se corriger, etc. Bien que le modelage permette aux stagiaires d'entamer une réflexion sur leurs procédures d'intervention (Ria et Serres, 2005), l'apprentissage de l'enseignement par l'imitation comporte également des limites, puisqu'il ressort que certains stagiaires observent les leçons de leur PEA sans chercher à en extraire le savoir pratique (Zanting, Verloop et Vermunt, 2003) et qu'il n'est pas, de toute façon, possible pour les stagiaires de reproduire en parfaite homologie les styles communicationnels et autres comportements et attitudes de leur PEA (Holvast et al., 1993). La capacité d'enseigner ne s'acquiert pas non plus indépendamment de la supervision reçue durant le stage de la part de la PEA (Brunelle et Brunelle, 1999). Au cours des prochains développements, nous montrerons combien la PEA est importante aux yeux du stagiaire et à quel point le travail qu'elle réalise auprès de lui peut l'influencer dans sa réussite comme dans son échec.

\section{Point de mire sur la personne enseignante associée (PEA)}

Aborder la question de la personne enseignante associée (PEA) s'impose naturellement lorsqu'il est question de formation pratique à l'enseignement. En effet, plusieurs études s'entendent pour dire que la PEA exerce une forte influence sur le développement professionnel du futur enseignant (Kramer-Hayon et Wubbels, 1993; McIntyre et Byrd, 1998; Mitchell et Schwager, 1993; Talvitie et al., 2000), qu'elle façonne les croyances et les pratiques de celui-ci (Hastings, 2004), qu'elle influence substantiellement le développement de ses dispositions face à l'enseignement (Talvitie et al., 2000). Contrairement à la personne superviseure universitaire (PSU) dont le rôle apparaît plus ambigu et la contribution à la réussite des stagiaires plus difficile à apprécier (Keller et Grossman, 1994; McIntyre et Killian, 1987; Mitchell et Schwager, 1993; Talvitie et al., 2000), la personne enseignante associée est vue comme celle qui peut se révéler un modèle à suivre et qui amène le stagiaire à devenir un enseignant. Le stagiaire tend à s'identifier à la PEA durant les stages (Legault, Charbonneau, Chevrier et Grégoire-Dugas, 1997). Ses commentaires et ses conseils tendent à être considérés comme plus utiles et plus facilitateurs que ceux provenant des formateurs universitaires ou superviseurs universitaires (Koster et al., 1998). 
Le travail de la PEA, plus particulièrement, s'inscrit dans des durées variables selon les programmes de formation concernés. Il consiste en un accompagnement au sens entendu par Koster et al. (1998) en ce qu'il implique pour un enseignant initié et plus chevronné de mener une action éducative auprès d'un collègue moins expérimenté en vue de promouvoir son développement sur les plans personnel et professionnel. À cette fin, la PEA médiatise la relation du stagiaire à la réalité du travail et agit à la manière d'un modèle, d'un enseignant ou d'un parrain qui encourage, conseille et soutient l'apprenti dans son projet de devenir enseignant. Ce travail en est aussi un de supervision au sens entendu par Villeneuve (1994) et par Brunelle, Drouin, Godbout et Tousignant (1988). En effet, il s'agit pour la PEA de planifier, de mettre en place et de réguler les conditions d'une analyse réflexive réussie autour de différents objets de travail à maîtriser se rapportant à l'enseignement, au développement des compétences ainsi qu'à l'autonomie professionnelle du stagiaire.

La fonction de PEA, toutefois, n'est pas toujours facile à exercer et peut, par moments, être une source de culpabilité, de frustration et d'anxiété. La culpabilité est particulièrement ressentie par les PEA lorsqu'elles ont l'impression de consacrer trop peu de temps aux stagiaires et lorsqu'elles observent un manque de progrès chez les premiers ainsi que chez les élèves qu'elles leur confient. De leur côté, les stagiaires rapportent de l'insatisfaction face à leur PEA s'ils ne reçoivent pas assez de rétroaction, si ce dernier est trop général, trop différé, trop négatif ou quand ils obtiennent des conseils qui s'avèrent improductifs pour eux (Mitchell et Schwager, 1993; Talvitie et al., 2000).

La culpabilité des personnes enseignantes associées peut se transformer en frustration quand celles-ci se sentent incapables de procurer à leur stagiaire le type de soutien qu'elles voudraient leur donner en raison des autres contraintes du travail. Cette frustration est encore plus fortement ressentie lorsque le manque de temps se traduit en fin de compte par une collaboration réduite avec les partenaires de l'université et par un manque d'informations, de connaissances ou d'expérience de leur côté. À certains égards, les stagiaires sont aussi des sources de frustrations pour les PEA, notamment ceux qui manifestent peu d'habiletés, peu d'émotions envers les élèves en classe, qui ne semblent pas vouloir s'investir avec assez d'intensité (Hastings, 2004) ou qui manquent d'éthique professionnelle.

La difficulté à composer avec le fait de jouer le double de rôle de mentor et d'évaluateur auprès du stagiaire peut créer de l'anxiété chez les PEA (Hastings, 2004), puisque cela peut interférer avec la création et le maintien d'une relation productive (Mozen, 2005) et affaiblir l'impact de l'accompagnement. Or, la qualité de cette relation peut être un stimulus invitant le stagiaire à se compromettre davantage et à accepter de délaisser des moyens d'enseignement plus familiers pour se centrer davantage sur l'apprentissage des élèves (Talvitie et al., 2000).

L'anxiété est également ressentie par les PEA lorsqu'elles sentent que leur jugement professionnel peut être remis en question par le superviseur universitaire au moment de l'évaluation des stagiaires (Hastings, 2004) et lorsqu'elles perçoivent leur crédibilité menacée par le manque de réussite de leur stagiaire. Sur ce dernier point, soulignons que l'échec en stage est rarement le fait d'un seul facteur (Schmidt et 
Knowles, 1994). Il s'agit la plupart du temps d'une combinaison malheureuse de plusieurs éléments, y compris le travail de la PEA auprès du stagiaire (Knowles et Sudzina, 1994). L'absence d'attentes claires, des changements de cap impromptus, l'incapacité de la part de la PEA de mettre en mots son savoir d'action et d'expliciter ses pratiques d'enseignement, l'insuffisance des rétroactions informant le stagiaire de l'effet qu'il a sur le groupe et l'absence d'une relation lui manifestant un soutien suffisant sont autant d'éléments relevant de problèmes de communication entre les deux acteurs qui peuvent affecter négativement la qualité de la supervision (KramerHayon et Wubbels, 1993), sinon mener à l'échec.

Il est important de souligner, par ailleurs, que les PEA sont rarement choisies en fonction de leurs habiletés en supervision, parce que ces dernières sont imprécises et difficiles à mesurer. De prime abord, de telles habiletés n'influent en rien sur les capacités des PEA à enseigner efficacement à des élèves. Cela signifie uniquement que les PEA ne possèdent pas, du simple fait qu'elles enseignent, les connaissances et les habiletés pour diriger la formation d'un adulte sur le terrain. L'insuffisance, voire l'absence de contrôle de la qualification, des compétences et des croyances de la PEA sur les façons de devenir enseignant représente indéniablement un facteur susceptible de limiter la portée éducative d'un stage. Ainsi, cette expérience peut créer de très importantes dissonances chez le stagiaire s'il se sent laissé à lui-même sans accompagnement de la part de la PEA. Son expérience peut s'avérer d'autant plus difficile qu'il perçoit une trop forte contradiction entre ce que véhicule son programme de formation et ce que prône sa PEA dans le milieu d'accueil, notamment si cette dernière réfute ou dénigre ce qui vient de l'établissement de formation. Un sentiment d'inconfort et d'incompétence pernicieux peut aussi l'habiter s'il doit vivre à répétition des situations difficiles pour lesquelles il n'est pas outillé. Rickard et Veal (1996) estiment ainsi qu'une PEA montrant un faible degré de compétence en matière de supervision pédagogique peut procurer des expériences beaucoup moins productives, voire néfastes au bon développement professionnel du stagiaire.

\section{Former les personnes enseignantes associées à la supervision pédagogique}

La préoccupation à l'égard de la formation des personnes enseignantes associées n'est pas récente. Elle a été longtemps et elle demeure l'un des aspects les plus négligés et l'un des plus potentiellement influents dans la formation des futurs enseignants (Keller et Grossman, 1994; Koster et al., 1998; McIntyre et Killian, 1987). Dans un document publié au milieu des années 1990, le ministère de l'Éducation du Québec (1995) a identifié neuf responsabilités de l'université au regard des stages, dont celle d'élaborer et de mettre en œuvre localement, en collaboration avec ses partenaires du milieu scolaire, une formation particulière pour la PEA affectée à l'encadrement pédagogique. La personne qui assume un tel rôle, reconnaît-il, "...aura besoin d'acquérir une compétence dans la supervision professionnelle dont les principaux aspects sont l'intervention auprès des stagiaires, l'analyse réflexive 
Encore aujourd'hui, le manque ou I'insuffisance de préparation des PEA à la supervision pédagogique demeure un constat global rapporté par la documentation scientifique en formation initiale en enseignement, y compris en éducation physique (Comité d'orientation de la formation du personnel enseignant [COFPE], 2005; Legault et al., 1997; Ouellet, 2002; Rickard et Veal, 1996). des pratiques pédagogiques et l'évaluation de la compétence professionnelle des stagiaires" (MEQ, 1995, p. 13). Le MEQ recommandait que cette capacité d'accompagner les stagiaires dans leur développement professionnel soit facilitée par la mise sur pied de programmes s'appuyant sur différents modèles et tenant compte des besoins des personnes enseignantes associés (PEA). Il souhaitait que les activités de formation, d'une durée totale variant entre 30 et 60 heures, soient réparties tout au long de la durée de l'encadrement des stagiaires.

Encore aujourd'hui, le manque ou l'insuffisance de préparation des PEA à la supervision pédagogique demeure un constat global rapporté par la documentation scientifique en formation initiale en enseignement, y compris en éducation physique (Comité d'orientation de la formation du personnel enseignant [COFPE], 2005; Legault et al., 1997; Ouellet, 2002; Rickard et Veal, 1996). Dans certains milieux, le fait d'avoir reçu une formation pèserait d'ailleurs assez peu dans le fait d'être recruté pour exercer cette fonction. Une étude publiée au Québec par Lacroix-Roy, Lessard et Garant en 2003 révèle en outre que 81,5\% des PEA ont reçu moins de 30 heures de formation en cette matière, la moyenne s'établissant à 14,8 heures de formation. Le nombre de stagiaires allant en augmentant, le manque de PEA conduit souvent à l'embauche d'enseignants en fonction de critères comme l'expérience en enseignement et la bonne volonté. Cette dernière s'inspire des motivations profondes comme de laisser sa formation en héritage, d'échanger avec un partenaire, de remettre en question sa pratique et de s'ouvrir aux nouveautés apportées par les stagiaires (Gervais et Desrosiers, 2005).

Des résultats de recherches montrent pourtant que la valeur des stages est accrue notamment quand les PEA ont suivi une formation à la supervision (McIntyre et Killian, 1987; OCDE, 2005), alors que des PEA peu ou mal formées en matière de supervision peuvent faire vivre des expériences d'apprentissage négatives à leur stagiaire (Rickard et Veal, 1996, p. 280). Ainsi, de notre point de vue, à défaut de la déplorer, il faut à tout le moins prendre acte de la tiédeur de la position du COFPE (2005) selon qui il faudrait envisager une obligation de compétence plutôt qu'une obligation de formation à proprement parler.

Selon la documentation consultée, l'accompagnement offert par les PEA présente plusieurs lacunes susceptibles d'affaiblir le processus d'apprentissage durant le stage. Par exemple, Koster et al. (1998) rapportent que les PEA ne sont pas toujours outillées pour réinvestir les concepts, les théories et les principes du champ de l'éducation dans leur travail auprès des stagiaires, ce qui ne permet pas d'assurer la continuité entre ce qui est enseigné dans le programme de formation et dans les milieux de stage (Mitchell et Schwager, 1993). Les PEA sont peu enclines à observer de manière soutenue et systématique les stagiaires lorsqu'ils enseignent et manifestent ou rapportent des difficultés à évaluer de manière critique et discriminante la qualité des pratiques d'enseignement (Keller et Grossman, 1994; Spallanzani, Sarrasin et Poirier, 1992; Spallanzani, Sarrasin et Goyette, 1995). L'évaluation sommative tout comme l'évaluation formative souvent requise par les partenaires universitaires ressortent comme des tâches exigeantes à leurs yeux. De surcroît, même lorsqu'elles parviennent à évaluer avec justesse les forces et les faiblesses des 
stagiaires, les PEA tendent à éviter les remarques critiques lorsque des faiblesses sont constatées et elles reconnaissent se sentir dépourvues quand vient le temps d'apporter de l'aide aux stagiaires pour pallier certaines difficultés précises.

Les personnes enseignantes associées en éducation physique estiment manquer de préparation pour remplir adéquatement leurs fonctions de supervision (Spallanzani et Sarrasin, 1994). Elles disent également éprouver de la difficulté à définir leurs besoins de formation. Ce n'est qu'avec le temps et de l'expérience qu'elles parviennent à se doter d'outils, de procédés, de moyens divers leur permettant d'accompagner les stagiaires dans leur développement professionnel. L'apprentissage de la supervision pédagogique passerait également par l'observation d'autres superviseurs (collègues enseignants ou superviseurs universitaires) ainsi que par leur propre expérience de stagiaire ayant pris part à un processus de supervision (Albuquerque, Graça, Januario et Durand, 2004). Les PEA tentent alors de reproduire ce qui leur a paru productif tout en évitant d'adopter certaines pratiques jugées non appropriées ou inefficaces (Rickard et Veal, 1996).

Les séances générales d'information ainsi que les ateliers de formation à la supervision de stagiaires sont fortement appréciés de la part des PEA (Koster et al., 1998; Spallanzani et al., 1992). Les étudiants qui effectuent leur stage auprès d'une PEA qui a été formée à la supervision ont l'impression d'être mieux dirigés que ceux qui font leur stage avec un intervenant non formé. Cela tend à confirmer la perception que ces occasions de formation peuvent influencer favorablement les pratiques de supervision des stagiaires (McIntyre et Byrd, 1998; McIntyre et Killian, 1987). Les habiletés de supervision comme la fréquence des interactions et la quantité de rétroaction donnée peuvent s'améliorer grâce à un système personnalisé de formation (Koster et al., 1998). Les PEA entraînées apprennent à devenir moins contrôlantes, à adopter un style plus indirect, plus axé sur les tâches que celui des PEA non entraînées (Rickard et Veal, 1996). En outre, il apparaît que les PEA formées à la supervision, bien qu'elles puissent devenir plus exigeantes, adoptent des attentes plus réalistes à l'égard de leurs stagiaires et semblent avoir plus confiance en leurs compétences comme superviseures. Les formations influenceraient aussi de façon positive l'encadrement d'un stage, la mise en place de processus clairs pour travailler avec les futurs maîtres et la définition des contextes d'apprentissage offerts au stagiaire (Boudreau et Baria, 1998; Koster et al., 1998). À l'instar des comportements d'enseignement des stagiaires, certains comportements de supervision peuvent changer à l'intérieur d'une période de dix semaines, c'est-à-dire la durée d'un stage (Hughley, 1973, cité dans Rickard et Veal, 1996). Toutefois, comme c'est le cas en formation à l'enseignement, un effet de lessivage («wash-out effect») serait également observable en formation à la supervision, cela ayant pour conséquence potentielle d'en limiter la portée (Boudreau et Baria, 1998). Ces indications ne manquent pas d'interroger la signifiance et l'efficacité des dispositifs de formation à la supervision utilisés par les PEA.

De manière générale, il semble qu'on se soit encore assez peu soucié de la définition du statut de la PEA sur les plans institutionnel et socioprofessionnel, de sa réelle incorporation au sein d'équipes de formateurs d'enseignants, de sa formation 
formelle, de l'évaluation de ses compétences et de sa sélection pour occuper cette fonction de premier plan au regard de la formation initiale (Chaliès et Durand, 2000; Koster et al., 1998; OCDE, 2005; Raymond, 2006; Rikard et Veal, 1996).

\section{Articuler les compétences à la supervision des personnes enseignantes associées et les fonctions qu'elles doivent remplir}

Par son action en matière de supervision, la personne enseignante associée doit viser le développement du stagiaire sur les plans personnel et professionnel (Keller et Grossman, 1994; Talvitie et al., 2000) puisque, comme l'affirment Pilon (2004) et Daele (2004), le développement professionnel ne peut s'opérer sans la transformation personnelle du praticien. Le développement professionnel est un processus complexe par lequel le futur enseignant modifie sa manière d'exercer sa pensée critique, de planifier et de mettre en œuvre la gestion de sa classe, de se percevoir et de comprendre ce que sont l'enseignement et l'apprentissage en contexte scolaire (Koster et al., 1998). Le développement professionnel se nourrit des interactions avec l'altérité. Il est lié à la construction ainsi qu'à la clarification de l'identité professionnelle et il a pour point de départ comme d'arrivée le développement de la maîtrise des pratiques professionnelles (Donnay et Charlier, cités dans Daele, 2004).

Gervais (1997) affirme que la supervision de stagiaires implique une expertise spécifique d'un autre ordre que celle d'enseigner. Devenir PEA, c'est adopter en parallèle une autre posture identitaire que celle de l'enseignant (COFPE, 2005) et accepter de modifier son rapport au savoir, de même qu'aux savoirs (Desbiens, Spallanzani, Brunelle, Turcotte et Roy, 2006b). Contrairement à la situation qui prévaut pour la formation initiale à l'enseignement au Québec, il n'y a pas encore, à notre connaissance, de référentiel formalisé identifiant et définissant les compétences attendues des PEA selon les ordres et les secteurs d'enseignement (Banville, 2002; COFPE, 2005), bien que des travaux soient activement menés en ce sens et fassent l'objet de discussions de haut niveau (Gervais, 2007). En outre, rappelons qu'au milieu des années 1990 la défunte Association québécoise universitaire en formation des maîtres (AQUFOM) a déployé des efforts pour bâtir un tel cadre de référence. Comme nous le verrons plus bas, des éléments communs à différents secteurs sont identifiables. Toutefois, l'enseignement de l'éducation physique recèle certaines spécificités (Carlier, 2002; Spallanzani et Sarrasin, 1994; Spallanzani, 2003) qui ne manquent pas de poser des défis sur le plan de la supervision. Insistons ainsi sur le fait que, dans cette matière scolaire, le stagiaire est appelé à enseigner plusieurs disciplines pour lesquelles son degré d'expertise peut varier considérablement. Selon la nature des activités et des tâches au programme, il peut, dans une même journée, devoir exploiter divers plateaux de travail, que ce soit à l'intérieur et à l'extérieur de l'école. Enfin, la nature même des objets expérimentés dans cette matière fait en sorte que le stagiaire doive gérer de manière sécuritaire des situations très ouvertes où l'environnement sociophysique est instable, plus difficilement prévisible et le mouvement 
humain omniprésent (Desbiens, Brunelle, Spallanzani et Roy, 2005).

Il nous semble que les efforts pour déterminer les compétences professionnelles que les PEA doivent développer en matière de supervision n'ont pas toujours eu pour point d'ancrage une réflexion formalisée et articulée portant sur les fonctions de la PEA, c'est-à-dire sur le rôle assumé par un ensemble d'actes de formation et par la supervision concourant à l'atteinte d'un but précis, et ce, afin de permettre à la PEA de s'adapter aux exigences de la situation de supervision dans laquelle elle est engagée. Si l'on peut facilement admettre que la PEA remplit une fonction particulière de l'enseignement par une aide de proximité, de compagnonnage (Carlier et Vandesande, 2004), sait-on ce qu'elle fait précisément et dans quels buts? Koster et al. (1998), par exemple, considèrent que la PEA remplit six fonctions auprès du stagiaire : l'orientation, le soutien personnel, la guidance, l'information notamment au fil des épisodes de rétroaction, la liaison entre le milieu de pratique et l'institution de formation des maîtres et, enfin, l'évaluation. Cette façon de cadrer le travail des PEA peut correspondre à de grandes catégories de leur activité effective, mais est-elle complète et entièrement satisfaisante? Ne devrait-on pas opérer des ajouts ou des substitutions? Il y a, nous semble-t-il, matière à réflexion sur ces aspects encore relativement peu traités.

Sans oublier ce qui précède, il apparaît raisonnable d'admettre que, d'un premier abord assez général, la PEA doit posséder une expertise technique, connaître le comportement humain, faire preuve de leadership, posséder de bonnes habiletés conceptuelles et organisationnelles (Bujold, 2002). De manière un peu étonnante, les faiblesses les plus marquées en matière de supervision résideraient dans la maîtrise des processus d'apprentissage, de même que dans celle des phénomènes relationnels (Bujold, 2002).

Pour leur part, Wiles et Bondi (2000) sont d'avis que la PEA devrait être capable de lire et d'interpréter le niveau de développement professionnel de l'enseignant de manière à déterminer ses besoins et à y répondre notamment par la mise en place de conditions d'apprentissage du métier, de même que par l'adoption de postures de supervision adaptées (Brunelle et Brunelle, 1999; Desbiens, Brunelle, Spallanzani et Roy, 2006a; Desbiens et al., 2006b). Ce point de vue conduit à penser les formations à la supervision non pas seulement en considération de techniques d'intervention comme l'observation, la rétroaction et le renforcement, toujours pertinentes par ailleurs (Jordan et al., 2004; Mozen, 2005), mais aussi en fonction de cadres conceptuels permettant de comprendre et de situer le développement professionnel des stagiaires en enseignement et leurs besoins.

La capacité de la PEA à inscrire le stagiaire dans une analyse réflexive de son action professionnelle apparaît centrale chez plusieurs auteurs (Brunelle et al., 1988; Brunelle et Brunelle, 1999; Carlier, 2002; Desbiens et al., 2006a, 2006b; MEQ, 1994; Mozen, 2005; Normand-Guérette, 1998). Il en est également ainsi en ce qui concerne la capacité à le motiver et à lui donner confiance (Carlier, 2002; Desbiens et al., 2006b), de même qu'en ce qui a trait à la capacité de l'évaluer et à documenter minutieusement ses progrès comme ses difficultés (Brunelle et al., 1988; Brunelle et Brunelle, 1999; Desbiens et al., 2006b; Koster et al., 1998; Keller et Grossman, 1994; 
MEQ, 1994; Mozen, 2005; Normand-Guérette, 1998).

Une attention particulière semble se dégager de la documentation consultée à propos de l'entretien de conseil pédagogique (conférence). Celui-ci survient à différents moments durant le stage. Il constitue une occasion où le stagiaire et la PEA discutent de l'expérience d'enseignement vécue par le premier, de ses progrès ou difficultés (Keller et Grossman, 1994; Mitchell et Schwager, 1993). Considéré comme une situation cruciale de formation professionnelle des enseignants, cet entretien a pour objet de créer un vocabulaire commun et d'aider le stagiaire à construire son expérience de l'enseignement (Bertone, 2004; Dugal, 2004). Il apparaît avant tout comme une modalité de formation centrée sur le soutien affectif des stagiaires dans laquelle les PEA privilégieraient des interactions visant la protection de ces derniers et la réduction de leur anxiété, au détriment de celles portant sur leurs erreurs et leurs difficultés. Durant les entretiens, une attention particulière serait accordée aux préoccupations des PEA et des stagiaires concernant la gestion du groupe d'élèves, du matériel, du temps et de l'espace aux dépens des aspects liés aux contenus d'enseignement (Dugal, 2004).

Les entretiens-conseils ne sont pas très fréquents (Banville, 2002). Il ne faut toutefois pas exclure que les PEA discutent de questions professionnelles avec les stagiaires à d'autres moments que lors d'entretiens formels. De manière générale, les stagiaires jouent un rôle plus effacé durant ces moments avec les PEA, surtout en début de stage. Leriche, Desbiens et Dugal (2008) rapportent que les stagiaires de leur étude occupaient une plus grande proportion du temps de parole à mesure que le stage avançait.

Les PEA sont peu portées à expliciter les connaissances et les croyances qui sous-tendent leur style d'enseignement. Zanting et al. (2003) croient que, si les PEA développaient cette capacité à rendre plus explicites des parcelles de leurs savoirs par l'entretien de conseil pédagogique, les stagiaires obtiendraient de l'information nouvelle, spécifique et pragmatique à propos de l'enseignement susceptible d'être réinvestie rapidement dans leur pratique. Cela leur permettrait d'accéder aux connaissances et aux croyances des PEA à propos de l'enseignement et à leurs pensées durant les phases pré-active, interactive et post-active. Ces cognitions dénotent les considérations et les préférences que les PEA peuvent avoir concernant l'enseignement et expliquent en partie pourquoi elles adoptent le style qu'elles manifestent. Les auteurs croient, enfin, que ces épisodes d'explicitation auraient pour effet de clarifier le sens donné par les stagiaires aux conseils et aux évaluations formulés par les PEA à l'égard notamment de leurs prestations d'enseignement.

\section{Pour conclure}

La supervision d'un stagiaire relève d'une expertise particulière qui fait appel à des capacités nombreuses. Certaines de ces capacités font l'objet d'une reconnaissance par plusieurs auteurs, mais la définition d'un référentiel de compétences en matière de supervision pédagogique devrait s'appuyer entre autres choses sur une 
réflexion approfondie à propos des fonctions exercées effectivement par la PEA dans les différentes phases du processus d'accompagnement du stagiaire.

La pratique supervisée de l'enseignement s'impose désormais comme un point d'ancrage pour le développement de compétences en enseignement. Le stage constitue un type d'expérience susceptible de transformer en profondeur les représentations du stagiaire quant au métier d'enseignant et au système scolaire ainsi que les perceptions qu'il a des élèves et de lui-même en tant qu'apprenant et intervenant. L'apprentissage de l'enseignement de l'éducation physique par l'intermédiaire du stage nécessite un engagement sérieux de la part du stagiaire sur les plans affectif et cognitif. Cependant, pour produire tous ses fruits, cette expérience doit être médiatisée par l'intervention de tierces personnes capables d'aider le stagiaire à élargir son répertoire de pratiques, à les analyser, à réaliser des prises de conscience, à exercer et à mesurer son jugement professionnel, etc. Parmi ces intervenants dont les actions sont porteuses de changements sur les plans professionnel et personnel, on trouve la PEA, qui reçoit le stagiaire dans son école et l'accompagne au quotidien, mais aussi tout un ensemble de formateurs (la direction d'établissement, la PSU, les professeurs d'université, les autres collègues enseignants, etc.) dont les actions sont, bien souvent, plutôt lâchement coordonnées. Comme le rappelle Daele (2004), l'enseignant est le premier responsable de son développement professionnel, mais celui-ci s'actualise d'autant mieux que le praticien évolue dans un environnement favorable qui permet entre autres choses des interactions avec l'altérité.

\section{Références bibliographiques}

ALBUQUERQUE, A., GRAÇA, A., JANUARIO, C. et DURAND, M. (2004). Comment les conseillers pédagogiques utilisent dans leur activité de formation leur expérience personnelle passée en tant que professeur stagiaire, dans M. Loquet et Y. Léziard (dir.), Cultures sportives et artistiques : Formalisation des savoirs professionnels. Pratiques, formations, recherches (p. 293-298). Rennes: Presses universitaires de Rennes II.

BANVILLE, D. (2002). Literature Review of Best Practices of Cooperating Teachers in the USA. Communication présentée à la First China-US Conference on Best Practices in Physical Education. Pékin, 16-19 juillet.

BERTONE, S. (2004). Dynamique des interactions en situation de conseil pédagogique et développement de l'activité professionnelle d'une enseignante novice d'éducation physique et son conseiller pédagogique, dans $\mathrm{M}$. Loquet et Y. Léziard (dir.), Cultures sportives et artistiques: formalisation des savoirs professionnels. Pratiques, formations, recherches (p. 287-291). Rennes: Presses universitaires de Rennes II. 
BORGES, C. et DESBIENS, J.-F. (2003). Conclusion, dans C. Borges et J.-F. Desbiens (dir.), Savoir, former et intervenir dans une éducation physique en changement (p. 223-242). Sherbrooke : Éditions du CRP.

BOUDREAU, P. et BARIA, A. (1998). La définition donnée par des enseignants associés de la supervision d'un stagiaire, dans D. Raymond et Y. Lenoir (dir.), Enseignants de métier et formation initiale (p. 141-154). Bruxelles : De Boeck Université.

BRAWDY, P. et BYRA, M. (1995). Supervision of Preservice Teachers during an Early Field Teaching Experience. Physical Educator, 52(3), 1-13.

BRUNELLE, J. et BRUNELLE, J.-P. (1999). Un système de supervision de la maîtrise des compétences de base dans l'enseignement de l'éducation physique et à la santé. Document de travail.

BRUNELLE, J., DROUIN, D., GODBOUT, P. et M. TOUSIGNANT (1988). La supervision de l'intervention en activité physique. Montréal: Gaëtan Morin.

BUJOLD, N. (2002). La supervision pédagogique. Vue d'ensemble, dans M. Boutet et N. Rousseau (dir.), Les enjeux de la supervision pédagogique des stages (p. 9-23). Sainte-Foy: Les Presses de l'Université Laval.

CARLIER, G. (2002). Superviser des stagiaires en éducation physique : balises pour une fonction en voie de professionnalisation. Avante, 8(1), 96-111.

CARLIER, G. et VANDESANDE, I. (2004). Maître de stage, c'est-à-dire? Ou comment des maîtres de stages définissent leur fonction, dans M. Loquet et Y. Léziard (dir.), Cultures sportives et artistiques : formalisation des savoirs professionnels. Pratiques, formations, recherches (p. 277-280). Rennes: Presses universitaires de Rennes II.

CHALIÈS, S. et DURAND, M. (2000). L'utilité discutée du tutorat en formation initiale des enseignants. Recherche et formation, 35, 145-180.

COMITÉ D'ORIENTATION DE LA FORMATION DU PERSONNEL ENSEIGNANT (COFPE). (2005). La formation en milieu de pratique. De nouveaux horizons à explorer. Avis au ministre. Québec: Gouvernement du Québec.

COMMISSION ROYALE D'ENQUÊTE SUR L'ENSEIGNEMENT DANS LA PROVINCE DE QUÉBEC (1963). Rapport de la Commission royale d'enquête sur l'enseignement dans la province de Québec. Rapport Parent. Québec.

CONSEIL SUPÉRIEUR DE L'ÉDUCATION (2004). Un nouveau souffle pour la profession enseignante : avis au ministère de l'Éducation. Québec : le Conseil.

DAELE, A. (2004). Développement professionnel des enseignants dans un contexte de participation à une communauté virtuelle: une étude exploratoire. Rapport de recherche, DES en sciences de l'éducation, Université catholique de Louvain, Belgique. 
DESBIENS, J.-F., BRUNELLE, J.-P., SPALLANZANI, C. et ROY, M. (2006a). Mejorar el acompañamiento de los pasantes en enseñanza en educación física a través de la formación para la supervisión de los profesores asociados. Un proyecto en vías de elaboración en la Facultad de Educación Física y Deportiva (FEFD) de la Universidad de Sherbrooke. Revista de Educación, 339 (janvier-avril), 339361. http://www.revistaeducacion.mec.es/re339_14.htm

DESBIENS, J.-F., SPALLANZANI, C., BRUNELLE, J.-P., TURCOTTE, S. et ROY, M. (2006b). Concevoir autrement la formation à l'accompagnement des stagiaires en enseignement de l'éducation physique et sportive (ÉPS) pour faire évoluer les pratiques de supervision. XXIII e congrès de l'AIPU, 15 au 18 mai 2006, Monastir, Tunisie.

DESBIENS, J.-F., BRUNELLE, J.-P., SPALLANZANI, C. et ROY, M. (2005). Former les personnes enseignantes associées (PEA) pour améliorer la supervision des stagiaires de la Faculté d'éducation physique et sportive (FEPS) de l'Université de Sherbrooke. Actes du $20^{\mathrm{e}}$ congrès international de l'Association internationale de pédagogie universitaire (AIPU) (CD-Rom). 27 au 30 mai. Sherbrooke : Université de Sherbrooke.

DUGAL, J.-P. (2004). Conseil et formation : recherche coopérative et savoirs professionnels des conseillers pédagogiques, dans M. Loquet et Y. Léziard (dir.), Cultures sportives et artistiques: Formalisation des savoirs professionnels. Pratiques, formations, recherches (p. 293-298). Rennes: Presses universitaires de Rennes II.

GAUTHIER, C., DESBIENS, J.-F,, MALO, A., MARTINEAU, S. et SIMARD, D. (1997). Pour une théorie de la pédagogie. Sainte-Foy: Les Presses de l'Université Laval.

GERVAIS, C. (2007). La formation en milieu de pratique: la préparation des superviseurs et des enseignants associés. Cadre de référence pour la formation des enseignants associés et des superviseurs universitaires. Communication présentée au Comité d'agrément des programmes de formation à l'enseignement, Montréal, 26 mars.

GERVAIS, C. (1997). Spécificité du rôle du superviseur universitaire, dans M. Tardif et H. Ziarko (dir.), Continuités et ruptures dans la formation des maîtres au Québec. Sainte-Foy: Les Presses de l’Université Laval.

GERVAIS, C. et DESROSIERS, P. (2005). L'école, lieu de formation d'enseignant. Questions et repères pour l'accompagnement de stagiaires. Québec: Les Presses de l'Université Laval.

HASTINGS, W. (2004). Emotions and the Practicum: the Cooperating Teachers' Perspective. Teachers and Teaching: Theory and Practice, 10(2), 135-148.

HOLVAST, A.-J., WUBBELS, Th. et BREKELMANS, M. (1993). Socialization in Student Teaching, dans Th. Wubbels et J. Levy (dir.), Do You Know What You Look Like? (p. 136-145). Londres: Falmer Press, 198 p. 
JORDAN, P., PHILLIPS, M. et BROWN, E. (2004). We Train Teachers: Why Not Supervisors and Mentors. Physical Educator, 61(4), 219-222.

KELLER, D.L. et GROSSMAN, J.A. (1994). A Model for Improving Preservice Teacher/Cooperating Teacher Diad. Educational Resources Information Center. ED 377181.

KNOWLES, G.J. et SUDZINA, M.R. (1994). Failure in Student/Practice Teaching: A Skeleton in the Teacher Education Closet. Communication présentée au congrès annuel des formateurs d'enseignants. Atlanta, GA, 12 au 15 février. ED 378159.

KOSNIK, C. (sous presse). It Is Not Just Practice: Conflicting Goals, Unclear Expectations, and Mixed Messages. Reconceptualizing the Place of Practice Teaching in Teacher Education Programs dans F. Benson et C. Riches (dir.), How Might Teacher Education Live Well in a Changing World? Zurich : Peter Lang.

KOSTER, B., KORTHAGEN, F.A.J. et WUBBELS, Th. (1998). European Journal of Teacher Education, 21(1), 75-89.

KRAMER-HAYON, L. et WUBBELS, Th. (1993). Supervisors' Interpersonal Behavior and Student Teachers' Satisfaction, dans Th. Wubbels et J. Levy (dir.), Do You Know What You Look Like? (p. 123-135). Londres: Falmer Press, 198 p.

LACROIX-ROY, F., LESSARD, M. et GARANT, C. (2003). Étude sur les programmes de formation à l'accompagnement des stagiaires. Québec : Table MEQ-universités.

LEGAULT, J.-P., CHARBONNEAU, B., CHEVRIER, J. et GRÉGOIRE-DUGAS, T. (1997). Les besoins de perfectionnement des enseignants associés : approche définitoire participative, dans M. Tardif et H. Ziarko (dir.), Continuités et ruptures dans la formation des maîtres au Québec (p. 90-108). Sainte-Foy: Les Presses de l'Université Laval.

LERICHE, J., DESBIENS, J.-F. et DUGAL, J.-P. (2008). L'accompagnement des stagiaires: un enjeu pour la formation des enseignants. Communication présentée au colloque de l'Association pour la recherche sur l'intervention en sports (ARIS), 14 au 17 mai 2008, Rodez (France).

MARTINET, M.-A., GAUTHIER, C. et RAYMOND, D. (2001). La formation à l'enseignement. Les orientations. Les compétences professionnelles. Québec : Ministère de l'Éducation du Québec.

MASCIOTRA, D. (2007). L'agir compétent: une approche situationnelle. Observatoire des réformes en éducation (ORE).

MCINTYRE, D.J. et BYRD, D.M. (1998). Supervision in Teacher Education, dans G.R. Firth et E.F. Pajak (dir.). Handbook of Research on School Supervision (p. 409-427). New York: MacMillan. 
MCINTYRE, D.J. et KILLIAN, J.E. (1987). The Influence of Supervisory Training for Cooperating Teachers on Preservice Teachers' Development during Early Field Experiences. Journal of Educational Research, 80(5), 277-282.

MINISTÈRE DE L'ÉDUCATION DU QUÉBEC (1995). La formation à l'enseignement. Les stages. Québec: Gouvernement du Québec.

MINISTÈRE DE L'ÉDUCATION DU QUÉBEC. (1994a). La formation à l'enseignement. Les stages. Québec: Gouvernement du Québec.

MINISTÈRE DE L'ÉDUCATION (1994b). La formation à l'éducation préscolaire et à l'enseignement primaire. Orientations et compétences attendues. Québec : Gouvernement du Québec.

MITCHELL, M.F. et SCHWAGER, S. (1993). Improving the Student Teaching Experience: Looking to the Research for Guidance. Physical Educator, 50(1), 31-38.

MOZEN, D. (2005). Best Practices of Effective Student-teacher Supervision Based on NASPE Standards. JOPERD, 76(6), 40-45.

MUNBY, H., RUSSELL, T. et MARTIN, A.K. (2001). Teachers' Knowledge and How It Develops, dans V. Richardson (dir.), Handbook of Research on Teaching ( $4^{\mathrm{e}}$ éd.) (p. 877-904). Washington, D.C. : American Educational Research Association.

NORMAND-GUÉRETTE, D. (1998). La formation des enseignants associés. Des stratégies pour mieux les outiller dans leurs rôles d'enseignants et de formateurs, dans D. Raymond et Y. Lenoir (dir.), Enseignants de métier et formation initiale (p. 103-122). Bruxelles: De Boeck Université.

Organisation pour la coopération et le développement économiques (OCDE). (2005). Le rôle crucial des enseignants. Politiques d'éducation et de formation. Attirer, former et retenir des enseignants de qualité. Paris : Éditions OCDE.

OUELLET, M. (2002). La supervision universitaire de stage en enseignement. Aux grands malaises, les grands remèdes, dans M. Boutet et N. Rousseau (dir.), Les enjeux de la supervision pédagogique des stages (p. 65-78). Sainte-Foy: Presses de l'Université du Québec.

PILON, J.-M. (2004). Une formation universitaire d'orientation praxéologique : démarche de développement professionnel et de transformation personnelle. Interactions, 8(2), 73-93.

RAYMOND, D. (2006). En formation à l'enseignement: des savoirs professionnels qui ont une longue histoire, dans Y. Lenoir et M.-H. Bouiller-Oudot (dir.), Savoirs professionnels et curriculum de formation (p. 233-261). Sainte-Foy: Les Presses de l'Université Laval.

RIA, L. et SERRE, G. (2005). Un dispositif de formation par procuration, confrontation et simulation. Les cahiers pédagogiques, 435 (septembre), 29-30. 
RIKARD, G.L. et VEAL, M.L. (1996). Cooperating Teachers: Insight into Their Preparation, Beliefs, and Practices. Journal of Teaching in Physical Education, $15,279-296$.

SCHMIDT, M. et KNOWLES, J.G. (1994). Four Women's Stories of Failure as Beginning Teachers. Communication présentée au congrès de l'AERA, Nouvelle-Orléans, LA, 4-10 avril. ED 375080.

SCHÖN, D. (1994). Le praticien réflexif. Montréal: Logiques.

SPALLANZANI, C. (2003). Les éducatrices et les éducateurs physiques : en éducation à part entière. Lettre de la Chaire de recherche du Canada et du Centre de recherche sur l'intervention éducative, 2(1), 1-2.

SPALLANZANI, C. et SARRASIN, J. (1994). Effets d'une démarche de réflexion sur la formation de maîtres associés. Actes du colloque international Enseignement supérieur : stratégies d'apprentissage appropriées (p. 357-371) tenu à Hull en août 1993. Hull : UQAH, Bureau des services à la collectivité.

SPALLANZANI, C., SARRASIN, J. et GOYETTE, R. (1995). La perception des enseignants à l'égard de leur rôle de formateur auprès de stagiaires universitaires. Actes du colloque de l'AQUFOM (p. 133-144). Montréal : Université McGill.

SPALLANZANI, C., SARRASIN, J. et POIRIER, A. (1992). Effets d'une formation minimale à la supervision de stagiaires en éducation physique. Revue des sciences de l'éducation, XVIII(3), 409-427.

TALVITIE, U., PELTOKALLIO, L. et MÄNNISTÖ, P. (2000). Student Teachers' Views about Their Relationships with University Supervisors, Cooperating Teachers and Peer Student Teachers. Scandinavian Journal of Educational Research, 44(1), 79-88.

TARDIF, M. (2006). Qu'est-ce que le savoir d'expérience en enseignement? Bulletin de la Haute école de pédagogie de Berne, du Jura et de Neuchâtel, 3 (juin), 13-16.

TARDIF, M. et LESSARD, C. (1999). Le travail enseignant au quotidien. Sainte-Foy: Les Presses de l'Université Laval.

THIBEAULT, J. (1994). Étude comparative d'un programme de mentorat et du programme québécois de probation des nouveaux enseignants. Mémoire de maîtrise. Trois-Rivières : Université du Québec à Trois-Rivières.

VILLENEUVE, L. (1994). L'encadrement du stage supervisé. Montréal : Saint-Martin.

WILES, J. et BONDI, J. (2000). Supervision. A Guide to Practice (5 ${ }^{\mathrm{e}}$ éd.). Columbus $(\mathrm{OH})$ : Merrill.

ZANTING, A., VERLOOP, N. et VERMUNT, J.D. (2003). How Do Student Teachers Elicit Their Mentor Teachers' Practical Knowledge? Teachers and Teaching: Theory and practice, 9(3), 197-211. 